REVISTAS DE LA FAHCE

Faculad de Hunvridads y Cenoirs de lo Educadion | tMU

क्र
Educación Física y Ciencia

ISSN: 1514-0105

ISSN: 2314-2561

revistaefyc@fahce.unlp.edu.ar

Universidad Nacional de La Plata

Argentina

\title{
Propuesta de evaluación de la condición física para población general: Batería Dickens
}

Farinola, Martin Gustav; Dardano, Paula Lucía; Maroni, Guillermo

Propuesta de evaluación de la condición física para población general: Batería Dickens

Educación Física y Ciencia, vol. 22, núm. 1, 2020

Universidad Nacional de La Plata, Argentina

Disponible en: http://www.redalyc.org/articulo.oa?id=439963095006

DOI: https://doi.org/10.24215/23142561e114

Esta obra está bajo una Licencia Creative Commons Atribución-NoComercial-Compartirlgual 4.0 Internacional. 


\title{
Propuesta de evaluación de la condición física para población general: Batería
} Dickens

\author{
Martin Gustav Farinola \\ Instituto Superior de Educación Física $N^{\circ} 2$ "Federico $W$. \\ Dickens", Argentina \\ martinfarinola@ymail.com \\ Paula Lucia Dardano \\ Instituto Superior de Educación Física N² "Federico W. \\ Dickens", Argentina \\ Guillermo Maroni \\ Instituto Superior de Educación Física $N^{\circ} 2$ "Federico $W$. \\ Dickens, Argentina
}

A proposal of assessment of physical fitness for general population: Dickens Battery

DOI: https://doi.org/10.24215/23142561e114

Redalyc: http://www.redalyc.org/articulo.oa? id $=439963095006$

\author{
Recepción: 07 Junio 2019
}

Aprobación: 06 Febrero 2020

\section{ReSUMEN:}

El objetivo es presentar una batería de pruebas suficientemente precisas y prácticas para evaluar la condición física en adultos argentinos de población general. Mediante una revisión no sistemática de la literatura se seleccionaron pruebas de campo suficientemente prácticas, precisas y con antecedentes de utilización en Argentina. Estas fueron Ir y volver $20 \mathrm{~m}$ para aptitud cardiorrespiratoria, Flexión de tronco modificado para flexibilidad y Prensión manual, Salto en largo y Extensiones de brazos en 30 segundos para fuerza muscular. Estas pruebas fueron administradas por especialistas a 410 estudiantes de educación física de 18 a 29,9 años de edad de ambos sexos pertenecientes a instancias iniciales y finales de la carrera para construir valores de referencia locales. Los datos fueron presentados según sexo y edad mediante percentiles, media y desvío estándar y se estudiaron las diferencias entre los grupos. Se encontraron en varones mayores niveles de fuerza y aptitud cardiorrespiratoria que en mujeres, mientras que no hubo diferencias entre sexos en flexibilidad. Algunas pruebas presentaron diferencias entre categorías de edad, pero estas diferencias no fueron regulares. La descripción de los resultados permitió presentar por primera vez valores de referencia de adultos argentinos para la evaluación de la condición física en población general.

Palabras clave: Condición física, Salud, Adultos, Población general, Evaluación.

\section{ABstract:}

The aim of this study is to present a set of sufficiently accurate and feasible tests to evaluate physical fitness in Argentinean adults of the general population. Through a non-systematic review of the literature, field tests were selected that were sufficiently feasible, accurate and with a history of use in Argentina. These tests were 20-m shuttle run for cardiorespiratory fitness, Modified seat and reach for flexibility and Handgrip, Standing broad jump and Pushups in 30 seconds for muscle strength. These tests were administered by specialists to 410 physical education students from 18 to 29.9 years of age of both sexes, the belong to the initial and final stages of the course so as to build local reference values. The data were presented according to sex and age using percentiles, mean and standard deviation and the differences between groups were studied. Higher levels of strength and cardiorespiratory fitness were found in males than in females, while there were no differences between sexes in flexibility. Some tests presented differences between age categories, but these differences were not regular. The description of the results allowed us to present for the first time reference values of Argentinean adults for the evaluation of physical fitness in the general population.

KEYWORDs: Physical fitness, Health, Adults, General population, Assessment. 


\section{INTRODUCCIÓN}

La condición física, o aptitud física, consiste en una serie de atributos o características que la gente tiene o alcanza y que se relacionan con la capacidad de realizar actividad física (Caspersen, Powell \& Christenson, 1985). Sin contradecirse con lo anterior, los mismos autores sostienen que ser físicamente apto permite "realizar las tareas diarias con vigor y consciencia, sin excesiva fatiga y con suficiente energía para disfrutar del tiempo libre y responder a situaciones imprevistas" (Caspersen, Powell \& Christenson, 1985 , p. 128). En esta última definición queda implícito que una condición física suficiente arroja la posibilidad de realizar las actividades físicas necesarias para la vida cotidiana y algunas más para la resolución de situaciones imprevistas que requieran de actividad física (correr, empujar, transportar objetos, subir escaleras, etc.).

Por otro lado se cree que durante los últimos siglos una transición desde un estilo de vida tradicional físicamente activo a uno urbano sedentario trajo consigo una disminución de la actividad física habitual y una consecuente disminución de la condición física en el común de las personas (Farinola, 2006; Rode \& Shephard, 1994). A su vez esta disminución de actividad física habitual y de aptitud física ha sido sugerida como uno de los agentes relacionados con el aumento de la incidencia de las enfermedades crónicas no transmisibles en numerosos países (Chakravarthy \& Booth, 2003). En efecto, durante las últimas décadas se ha encontrado que existe una relación positiva entre condición física y salud (Blair et al., 1989; Blair et al., 1995; Katzmarzyk, Church \& Blair, 2004; Warburton, Gledhill \& Quinney, 2001; Warburton, Nicol \& Bredin, 2006). Esto reforzó el concepto de condición física relacionada a la salud (CFRS) que había empezado a sugerirse en la década de 1970 (Pate, 1988) y cuya definición incluye a la capacidad de llevar adelante las actividades físicas de la vida cotidiana pero ahora también a la reducción del riesgo de desarrollar prematuramente enfermedades relacionadas a un estilo de vida hipocinético (Bouchard, Blair \& Haskell, 2007; Pangrazi \& Hastad, 1989; Pate, 1988; Suni, Marjo \& Husu, 2009). En Argentina estas enfermedades son la principal causa de mortalidad (Ministerio de Salud, 2014), por lo tanto, desde el punto de vista de la salud, se vuelve relevante estudiar tanto la actividad física como la condición física y tender hacia niveles saludables.

Los beneficios de contar con una condición física saludable son variados y se relacionan con diferentes aspectos de la condición física (Garber et al., 2011). Estos múltiples aspectos son llamados capacidades físicas o dimensiones de la condición física. En general las dimensiones que forman parte de la CFRS se reconoce que son: la aptitud cardiorrespiratoria, la fuerza y resistencia muscular, la flexibilidad, la composición corporal (Kaminsky, 2010; Pescatello, 2014) y el componente neuromotor (Garber et al., 2011).

Si se tiene la intención de conducir a las personas hacia un nivel saludable de condición física surge la necesidad de evaluarla. Esta evaluación de la condición física resulta útil para: i) conocer el nivel inicial de la condición física de los alumnos en el marco de un proceso de acondicionamiento físico; ii) conocer la efectividad de las intervenciones docentes en dicho proceso; iii) eventualmente motivar a los alumnos al mostrarles las mejoras conseguidas; iv) describir el nivel de condición física de una población o grupo de poblaciones con fines diversos; entre otras (Blázquez Sánchez, 1992; George, Fisher \& Vehrs, 1996).

Creemos que las herramientas con las que se cuenta hoy para realizar esta tarea con adultos no deportistas en Argentina no son las óptimas, principalmente por la falta de valores de referencia adecuados. En el mejor de los casos se utilizan pruebas o test de calidad pero con valores de referencia de otros países y de otras realidades geográficas y culturales. Esta carencia de herramientas óptimas para la evaluación de la condición física en adultos de población general argentina es la que nos ha llevado a comenzar a desarrollar lo que denominamos Batería Dickens.

El objetivo de este trabajo es presentar la versión 1 de esta batería que consiste, por un lado, en la selección de un conjunto de pruebas suficientemente precisas y prácticas para evaluar algunas de las dimensiones que forman parte de la CFRS en adultos de población general y, por el otro, en la confección de valores de referencia normativos de adultos argentinos de ambos sexos de entre 18 y 29 años de edad para estas pruebas. 


\section{Metodología}

\section{Selección de las pruebas}

Se realizará una revisión no sistemática de las baterías vigentes en diferentes países e instituciones para adultos de población general en busca de test o pruebas que se sugieran utilizar internacionalmente para la evaluación de las dimensiones de la CFRS. Se optará por aquellas pruebas que reúnan evidencia de precisión (validez y confiabilidad) y que por su practicidad y antecedentes de uso en el medio local sean factibles de administrarse en entornos de campo por la mayoría de los profesionales del ejercicio.

\section{Valores normativos de referencia}

La muestra de referencia se conformó con sujetos de ambos sexos que se encontraron comenzando el primer año (ingresantes) y cursando el cuarto y último año (egresantes) de la carrera de Educación Física en el ISEF N² "Federico W. Dickens" entre los años 2014 y 2017. Se tomaron alumnos de ambos extremos de la carrera para obtener mayor variedad en cuanto a edades y niveles de condición física ya que se ha visto que los niveles de condición física pueden variar a lo largo del cursado de esta carrera (Delgado, Renda, Rojas \& Supital, 2011; Rojas, Renda, Cova \& Mostovoi, 2010; Vásquez-Gómez, Castillo-Retamal, Souza de Carvalho, Faundez-Casanova \& Torrealba-Campos, 2018). Para evitar posibles sesgos, de este grupo de alumnos se buscó que participe la totalidad, por lo tanto no fue necesario realizar una selección.

Para participar del estudio los sujetos debían tener entre 18 y 29 años de edad al momento de realizar las pruebas y poseer el certificado de salud para la práctica de actividades físicas emitido por profesionales médicos. Los sujetos fueron informados de los objetivos de las pruebas y su participación fue voluntaria y anónima. A estos sujetos se les administraron las pruebas seleccionadas en orden aleatorio permitiéndose la recuperación entre cada una de ellas. La prueba de aptitud cardiorrespiratoria se administró en día separado del resto de las pruebas. La administración de las pruebas fue realizada por profesores de educación física especializados en la materia.

\section{Tratamiento de datos}

Para la presentación de los resultados los sujetos serán divididos en categorías de sexo (masculino y femenino) y edad (18 a 19,9 y 20 a 29,9 años de edad). Para la descripción de los resultados se mostrará la distribución de las variables a través de diagramas de caja y se identificará si existen diferencias significativas entre los grupos a través de la prueba no paramétrica $U$ de Mann-Whitney estableciéndose el nivel de significancia estadística en 0,05. Los valores de referencia serán presentados a través de percentiles, promedio y desvío estándar de cada grupo de sexo y edad en cada prueba. El tratamiento de los datos se realizará con el programa IBM SPSS versión 20.

\section{RESULTADOS Y DiscusióN}

\section{Selección de pruebas}

En la tabla 1 se muestran las dimensiones de la condición física y las pruebas de campo utilizadas por diferentes baterías internacionales dirigidas a adultos de población general y por la Batería Dickens. 
TABLA 1

Dimensiones de la condición física y pruebas de campo propuestas en la Batería

Dickens y en diferentes baterías internacionales para adultos de población general

\begin{tabular}{|c|c|c|c|c|c|c|}
\hline Dimensión & Prueba & $\begin{array}{l}\text { Batería } \\
\text { ACSM }^{1}\end{array}$ & $\begin{array}{c}\text { Batería } \\
\text { ALPHA- } \\
\text { FIT }^{2}\end{array}$ & $\begin{array}{l}\text { Batería } \\
\text { CSEP- } \\
\text { PATH }^{3}\end{array}$ & $\begin{array}{l}\text { Batería } \\
\text { AFISAL- } \\
\text { INEFC }^{4}\end{array}$ & $\begin{array}{l}\text { Batería } \\
\text { Dickens }\end{array}$ \\
\hline \multirow{8}{*}{$\begin{array}{l}\text { Aptitud } \\
\text { cardiorrespiratoria }\end{array}$} & Caminar $2 \mathrm{~km}$ & & $\checkmark$ & & $\checkmark$ & \\
\hline & Variantes del test del escalón & $\checkmark$ & & $\checkmark$ & & \\
\hline & Carrera de 1,5 millas & $\checkmark$ & & & & \\
\hline & Caminar/correr $12 \mathrm{~min}$ (Cooper) & $\checkmark$ & & & & \\
\hline & Caminar 1 milla (Rockport) & $\checkmark$ & & $\checkmark$ & & $\sqrt{*}$ \\
\hline & YMCA en cicloergómetro & & & $\checkmark$ & & \\
\hline & Course Navette & & & & & $\checkmark$ \\
\hline & $\begin{array}{l}\text { Caminata submáxima en cinta } \\
\text { rodante (Ebbeling) }\end{array}$ & & & $\checkmark$ & & \\
\hline \multirow[t]{8}{*}{ Aptitud muscular ${ }^{5}$} & Prensión manual (Handgrip) & $\checkmark$ & $\checkmark$ & $\checkmark$ & $\checkmark$ & $\checkmark$ \\
\hline & 1 repetición máxima (1 RM) & $\checkmark$ & & & & \\
\hline & $\begin{array}{l}\text { Abdominales con cadencia } \\
25 / \mathrm{min} \text { (Curl-ups) }\end{array}$ & $\checkmark$ & & & $\checkmark$ & $\sqrt{ } *$ \\
\hline & $\begin{array}{l}\text { Variantes de extensiones de } \\
\text { brazos (Push-ups) }\end{array}$ & $\checkmark$ & $\checkmark$ & $\checkmark$ & & $\checkmark$ \\
\hline & $\begin{array}{l}\text { Press plano con cadencia } 30 \mathrm{~min} \\
\text { (YMCA) }\end{array}$ & $\checkmark$ & & & & \\
\hline & Salto vertical & & $\checkmark$ & $\checkmark$ & $\checkmark$ & \\
\hline & Salto horizontal & & & & & $\checkmark$ \\
\hline & Extensiones de tronco & & & $\checkmark$ & & \\
\hline \multirow[t]{2}{*}{ Flexibilidad } & $\begin{array}{l}\text { Flexión de tronco (Sit and } \\
\text { reach/(regla fija) }\end{array}$ & $\checkmark$ & & $\checkmark$ & & \\
\hline & $\begin{array}{l}\text { Flexión de tronco modificado } \\
\text { (regla móvil) }\end{array}$ & & & & $\checkmark$ & $\checkmark$ \\
\hline \multirow[t]{2}{*}{ Equilibrio } & Pararse en una pierna & & $\checkmark$ & $\checkmark$ & & $\sqrt{*}$ \\
\hline & Pararse en una pierna sin visión & & & & $\checkmark$ & \\
\hline \multirow{6}{*}{$\begin{array}{l}\text { Composición } \\
\text { corporal }\end{array}$} & Índice de masa corporal & $\checkmark$ & $\checkmark$ & $\checkmark$ & $\checkmark$ & $\sqrt{*}$ \\
\hline & Circunferencia de cintura & & $\checkmark$ & $\checkmark$ & & $\sqrt{*}$ \\
\hline & Índice de cintura/cadera & $\checkmark$ & & & $\checkmark$ & \\
\hline & Suma de pliegues & & & & & \\
\hline & $\begin{array}{l}\text { \% grasa corporal } \\
\text { (antropometría) }\end{array}$ & $\checkmark$ & & & $\checkmark$ & \\
\hline & $\begin{array}{l}\% \text { grasa corporal } \\
\text { (bioimpedancia) }\end{array}$ & $\checkmark$ & & & & \\
\hline
\end{tabular}

1 American College of sport Medicine (Kaminsky, 2010)

2 ssessing levels of physical activity and fitness (Suni et al., 2009)

3 Canadian Society for Exercise Physiology-Physical Activity Training for Health (CSEP, 2013)

4 Actividad Física y Salud para Adultos-Institut Nacional d'Educació Física de Catalunya (Rodríguez et al., 1998a)

5 "Aptitud muscular" es un concepto genérico que contiene a la fuerza máxima,

la potencia muscular y a la resistencia muscular localizada (Smith et al., 2014)

* Se encuentran en desarrollo y formarán parte de las sucesivas versiones de la Batería Dickens.

Puede observarse que no existe un conjunto de pruebas uniforme de uso internacional que se sugiera aplicar en los países. Por este motivo las pruebas seleccionadas para formar parte de la Batería Dickens fueron aquellas que, a la vez que contaron con evidencia suficiente de validez, confiabilidad y practicidad, tuvieron mayor tradición en nuestra institución y en el medio local.

Para el componente cardiorrespiratorio se escogió la prueba de Ir y volver $20 \mathrm{~m}$ utilizada recientemente en población escolar argentina (Aquila Dumit, 2012; Secchi, García, España-Romero \& Castro-Piñero, 2014). Para su administración sólo se necesita una superficie plana de 22 metros de longitud (20 para la ejecución 
de la prueba y 1 metro más en cada extremo por seguridad) y 1 metro de ancho por persona, lo que permite que el test pueda ser ejecutado por varios sujetos simultáneamente y en espacios reducidos. Esta característica lo vuelve más práctico al no necesitar una pista o campo para su administración. Por otro lado, y al ser un test incremental y máximo voluntario, se comienza a un ritmo suave y se finaliza cuando el participante se considere fatigado. Esto hace que personas de prácticamente cualquier nivel de aptitud física lo pueda ejecutar y además que se disminuya el riesgo de sobreexigencias. La validez con respecto al consumo máximo de oxígeno (VO.max) medido en forma directa resultó ser aceptable a buena en ambos sexos tanto en niños y adolescentes de 8 a 19 años $\left(\mathrm{r}=0,71 ; \mathrm{EEE}=5,9 \mathrm{ml} \cdot \mathrm{kg}^{-1} \cdot \mathrm{min}^{-1} ; \mathrm{n}=188\right)$ como en adultos de 18 a 50 años $(\mathrm{r}$ $=0,90 ; \mathrm{EEE}=4,7 \mathrm{ml} \cdot \mathrm{kg}^{-1} \cdot \mathrm{min}^{-1} ; \mathrm{n}=77$ ) (Léger, Mercier, Gadoury \& Lambert, 1988). Estudios posteriores corroboraron la elevada correlación entre el resultado en el Ir y volver $20 \mathrm{~m}$ y el VO.max en estudiantes de Educación Física de ambos sexos ( $\mathrm{r}=0,87 ; \mathrm{n}=25$ varones y 23 mujeres) (Paradisis et al., 2014). La confiabilidad test-retest (con una semana de diferencia) resultó ser buena a muy buena en ambos sexos tanto en los jóvenes $(\mathrm{r}=0,89 ; \mathrm{n}=139)$ como en los adultos $(\mathrm{r}=0,95 ; \mathrm{n}=81)$, no encontrándose diferencias significativas entre el test y el re test (Léger et al., 1988).

Para la aptitud muscular se escogieron las pruebas Prensión manual, Extensiones de brazos en 30 seg y Salto horizontal. Prensión manual con dinamómetro es una prueba máxima e isométrica para la evaluación de la fuerza muscular. Hasta nuestro conocimiento esta prueba no forma parte de ninguna batería Argentina pero se encuentra presente en todas las baterías internacionales revisadas (ver tabla 1). Es una prueba relativamente práctica debido a que, si bien se requiere de un instrumento específico (dinamómetro manual), el procedimiento es sumamente sencillo, seguro y requiere de poco tiempo. Además de esta practicidad Prensión manual cuenta con otras propiedades que la hacen una conveniente: correlaciona inversamente con la edad durante la adultez, correlaciona bien con la fuerza isométrica del tren inferior $(r=0,70$ a 0,72$)$ y resulta eficaz en la identificación de una baja capacidad de desplazarse (Lauretani et al., 2003). En Batería Dickens se utilizó un dinamómetro hidráulico marca North Coast Medical. Según informa el fabricante este dinamómetro cuenta con un coeficiente de correlación de 0,9999 al comparar su resultado con el de pesos conocidos, similar a lo obtenido con el dinamómetro hidráulico Jamar que es el instrumento más ampliamente citado en la literatura (Roberts et al., 2011). El dinamómetro Jamar obtuvo un coeficiente de correlación de 0,9994 al comparar su resultado con pesos conocidos (validez) y un coeficiente de correlación intraclase de entre 0,90 y 0,97 cuando se estudió su confiabilidad a través de un estudio test-retest (Mathiowetz, 2002).

En la prueba de Extensiones de brazos en 30 seg se debe realizar la mayor cantidad de extensiones de brazos correctas en 30 segundos. Los varones realizan la prueba con el punto de giro en el apoyo de pies y las mujeres en el apoyo de rodillas. Una versión similar de esta prueba fue utilizada en la batería bonaerense Pediafys (Barbieri, 1997). Es un test con alta practicidad debido al escaso costo de los materiales que se necesitan: un cronómetro y una colchoneta. No hemos encontrado estudios de validez para la prueba de Extensiones de brazos en $30 \mathrm{seg}$ en adultos de población general pero sí de pruebas similares que han tenido un resultado favorable (Baumgartner, Oh, Chung \& Hales, 2002). Los estudios disponibles son consistentes con una validez aceptable de esta prueba aunque esto necesita corroborarse. En cuanto a la confiabilidad de la prueba hemos realizado en nuestro laboratorio un estudio de correlación test-retest con una semana de diferencia entre cada intento en una muestra de 37 mujeres y 33 varones todos estudiantes de la carrera de Profesorado de Educación Física de nuestro instituto. El coeficiente de correlación intraclase ha resultado ser de 0,94 para las mujeres $(p<0,001)$ y de 0,89 para los varones $(p<0,001)$ no habiendo diferencias significativas entre el test y el retest tanto en el grupo de mujeres como en el de varones (prueba t de Student para muestras relacionadas $\mathrm{p}>0,05)$. Por lo tanto se acepta la confiabilidad de la prueba.

En la prueba de Salto horizontal se busca alcanzar la mayor distancia horizontal posible a través de un salto con ambas extremidades inferiores en simultáneo para poder evaluar la fuerza explosiva de estas extremidades. Esta prueba fue incluida en diversas baterías para adolescentes argentinos (Aquila Dumit, 2012; Barbieri, 
1997; Secchi et al., 2014). Para analizar su validez, García Lopez y Herrero Alonso (2005) midieron las variables fuerza-tiempo $(\mathrm{f}-\mathrm{t})$, velocidad-tiempo $(\mathrm{v}-\mathrm{t})$ y potencia-tiempo $(\mathrm{P}-\mathrm{t})$. En este trabajo se estudiaron 144 estudiantes de Educación Física ( 96 hombres y 48 mujeres) que realizaron 3 saltos verticales (SV) sobre plataforma de contacto y 3 saltos horizontales $(\mathrm{SH})$ sobre plataforma de fuerza. Se obtuvieron correlaciones significativas $(\mathrm{p}<0,05)$ entre $\mathrm{SH}$ y $\mathrm{f}-\mathrm{t}, \mathrm{SH}$ y $\mathrm{P}-\mathrm{t}$ (relativos al peso corporal) y SH y v-t. Paralelamente se obtuvieron diferencias significativas $(\mathrm{p}<0,05)$ en estas variables entre hombres y mujeres $(6-36 \%)$. También se obtuvieron altas asociaciones $(\mathrm{p}<0,001)$ entre $\mathrm{SH}$ y $\mathrm{SV}$ en hombres y en mujeres $(\mathrm{r}=0,68$ y $\mathrm{r}=0,69$, respectivamente). Según los autores de este trabajo, estos resultados muestran que el test de Salto horizontal es válido para evaluar la fuerza explosiva en extremidades inferiores (García López \& Herrero Alonso, 2005). Por otro lado, la confiabilidad del test se puso a prueba en otro estudio que involucró a 12 varones estudiantes de Educación Física. Para ello se llevó a cabo un trabajo en el que se realizó test-retest en un primer día arrojando un coeficiente de correlación de $0,97(\mathrm{p}<0,001)$. En un segundo día con una semana de diferencia, el test-retest arrojó un coeficiente de correlación de 0,99 ( $\mathrm{p}<0,001)$. Esta mínima mejora en el día 2 sugiere que el Salto horizontal es un test conocido y practicado por los sujetos, que está más automatizado y por tanto cada intento parece haber sido máximo. Mientras que en día 1 versus día 2 el coeficiente de correlación fue de 0,75 ( $\mathrm{p}<0,05)$. Según los autores, a partir de estos resultados, la confiabilidad de esta prueba se considera muy buena (López Elvira, Grande Rodríguez, Meana Riera \& Aguado Jódar, 1999).

Para la evaluación de la flexibilidad se escogió la prueba de Flexión de tronco modificado (FTM) con regla móvil. Una versión similar de esta prueba (con regla fija) fue utilizada en la batería bonaerense Pediafys para niños y adolescentes (Barbieri, 1997). La validez de las distintas versiones del test de flexión de tronco fue estudiada a través de un meta análisis tomando como criterio la extensibilidad de los músculos isquiosurales y lumbares (Mayorga-Vega, Merino-Marban \& Viciana, 2014). De este meta-análisis tomaremos sólo los resultados de la versión Modificada con regla móvil. Esta versión es la que se utiliza en la Batería Dickens y toma en consideración el largo de miembros inferiores y superiores para el establecimiento del resultado (Hoeger \& Hoeger, 2010). Los coeficientes de correlación entre FTM y extensibilidad de isquiosurales presentaron un rango de valores que van de 0,41 a 0,76 para varones y de 0,26 a 0,73 para mujeres. Para el caso de utilizar como criterio la extensibilidad lumbar estos valores estuvieron entre 0,21 y 0,40 en varones y entre 0,22 y 0,26 en mujeres. La conclusión de los autores es que en conjunto las distintas versiones del test de flexión de tronco, incluido el FTM, demostraron tener una validez moderada con respecto a la extensibilidad isquiosural y una validez baja con respecto a la extensibilidad lumbar. Por lo tanto esta prueba es un mejor indicador de la extensibilidad isquiosural que de la extensibilidad lumbar. La confiabilidad de las pruebas de flexión de tronco, en sus diferentes versiones fue revisada por Ayala, Sainz de Baranda, de Ste Croix y Santonja (2012). Estos autores concluyeron que las pruebas de flexión de tronco han demostrado poseer de forma generalizada una elevada confiabilidad, medida a través del índice de correlación intraclase, con valores en torno a 0,89-0,99 independientemente del sexo y del protocolo utilizado. En esta revisión incluyeron un trabajo que estudió la confiabilidad de la versión FTM encontrándose una correlación de 0,97 y 0,91 para hombres y mujeres adultos respectivamente.

\section{Resultados de las pruebas}

El porcentaje de participación de los sujetos fue del 98\% para ingresantes y del 92\% para egresantes. La causa principal de no participación fue la presencia una lesión o problema de salud anterior a la participación de los testeos. En ningún caso tuvo que abandonarse alguna prueba antes de cumplirse el criterio de finalización de la misma.

Las figuras 1, 2, 3, 4 y 5 muestran la distribución de los resultados en cada prueba para cada categoría de sexo y edad. Existieron diferencias estadísticamente significativas entre ambos sexos en todas las dimensiones testeadas excepto en flexibilidad. En cuanto a las categorías de edad existieron diferencias estadísticamente 
significativas entre ellas en cuatro dimensiones en el grupo de varones y en dos dimensiones en el grupo de mujeres (ver figuras 1 a 5 ).

Las tablas 2, 3, 4, 5 y 6 muestran los valores de referencia para cada prueba según categorías de sexo y edad.

\section{Discusión}

La presente batería es un primer intento de cubrir una vacancia de información relevante en Argentina para la promoción de la actividad física en general y para el campo del acondicionamiento físico en población general en particular. Este primer intento cuenta con virtudes y limitaciones.

Dentro de las virtudes podemos destacar que es la primera batería de estas características en la ciudad de Buenos Aires y hasta nuestro conocimiento en Argentina. Sus dos características principales son por un lado que utiliza pruebas válidas, confiables, prácticas y con antecedentes de utilización en Argentina, y por otro lado que presenta valores de referencia de sujetos adultos argentinos.

Con respecto a las pruebas seleccionadas se encontró que no existe hasta la fecha un conjunto de pruebas estandarizado de uso internacional que se aplique en los países para la evaluación de la CFRS en adultos de población general (ver tabla 1). Una característica que sí tienen en común las baterías disponibles es que las pruebas seleccionadas valoran solamente las dimensiones de la CFRS y cuentan con evidencia de validez y confiabilidad suficiente. Creemos que también es el caso de las pruebas elegidas para formar parte de la Batería Dickens. En todos los casos los estadísticos que permiten evaluar la precisión de las pruebas (validez y confiabilidad) arrojaron valores moderados a altos, suficientes por tratarse de pruebas indirectas de administración sencilla. Esto último, la sencillez o practicidad de las pruebas, es un requisito necesario para las pruebas de campo ya que su propósito es que puedan aplicarse sin mayores impedimentos en diversidad de situaciones y de contextos. 
Martin Gustav Farinola, et al. Propuesta de evaluación de la condición física para población gene...

\section{FIGURA 1}

Distribución de resultados en la prueba de Ir y volver $20 \mathrm{~m}$ (palieres completados) según sexo y edad

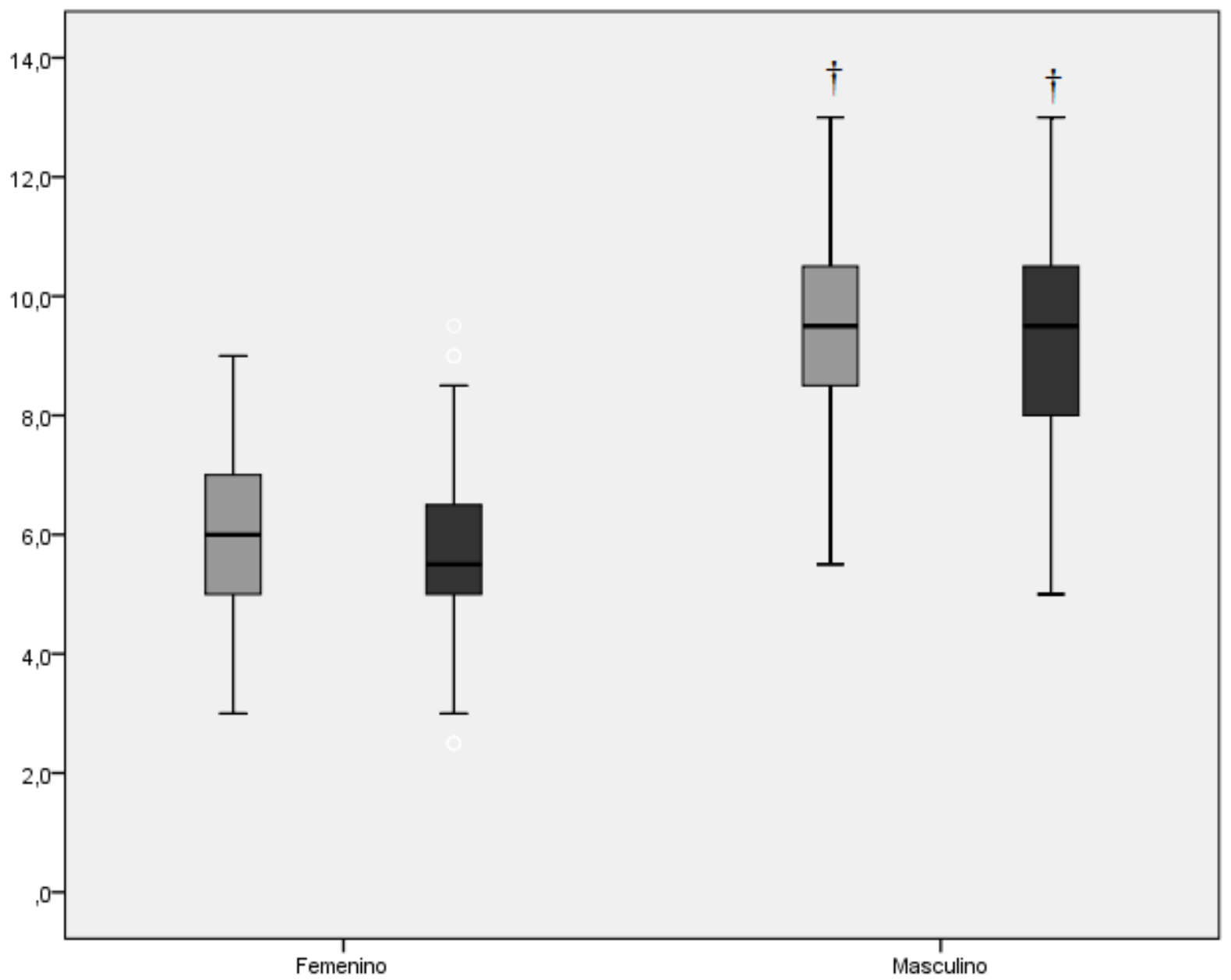

Cajas claras 18 a 19,9 años de edad, cajas oscuras 20 a 29,9 años de edad. †Diferencias significativas con mujeres de la misma edad $(\mathrm{p}<0,05)$ Fuente: elaboración propia. 
FIGURA 2

Distribución de resultados en la prueba de Flexión de tronco al frente modificado $(\mathrm{cm})$ según sexo y edad

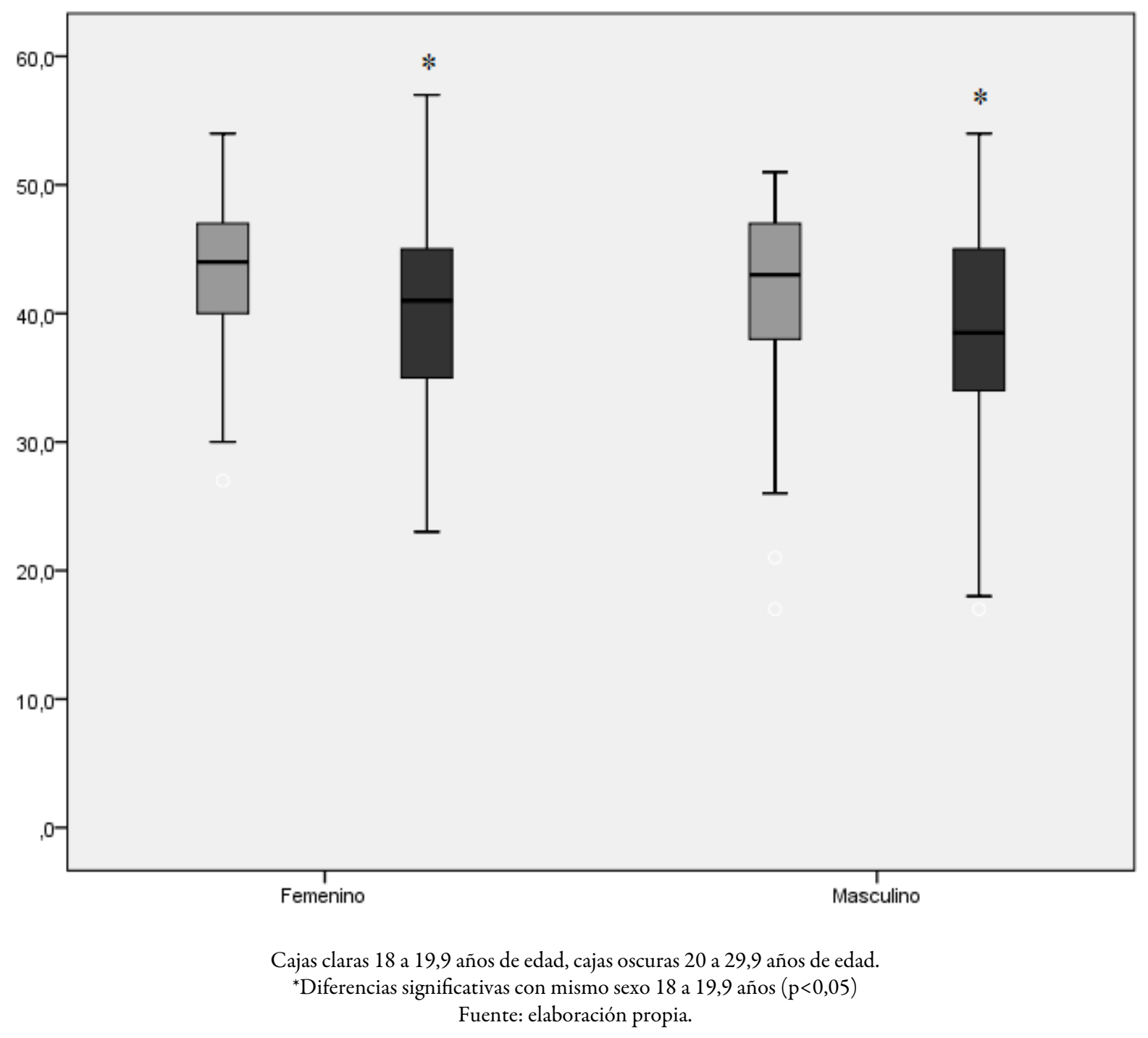


Martin Gustav Farinola, et al. Propuesta de evaluación de la condición física para población gene...

FIGURA 3

Distribución de resultados en la prueba de Prensión manual ( $\mathrm{kg}$, suma de ambas manos) según sexo y edad

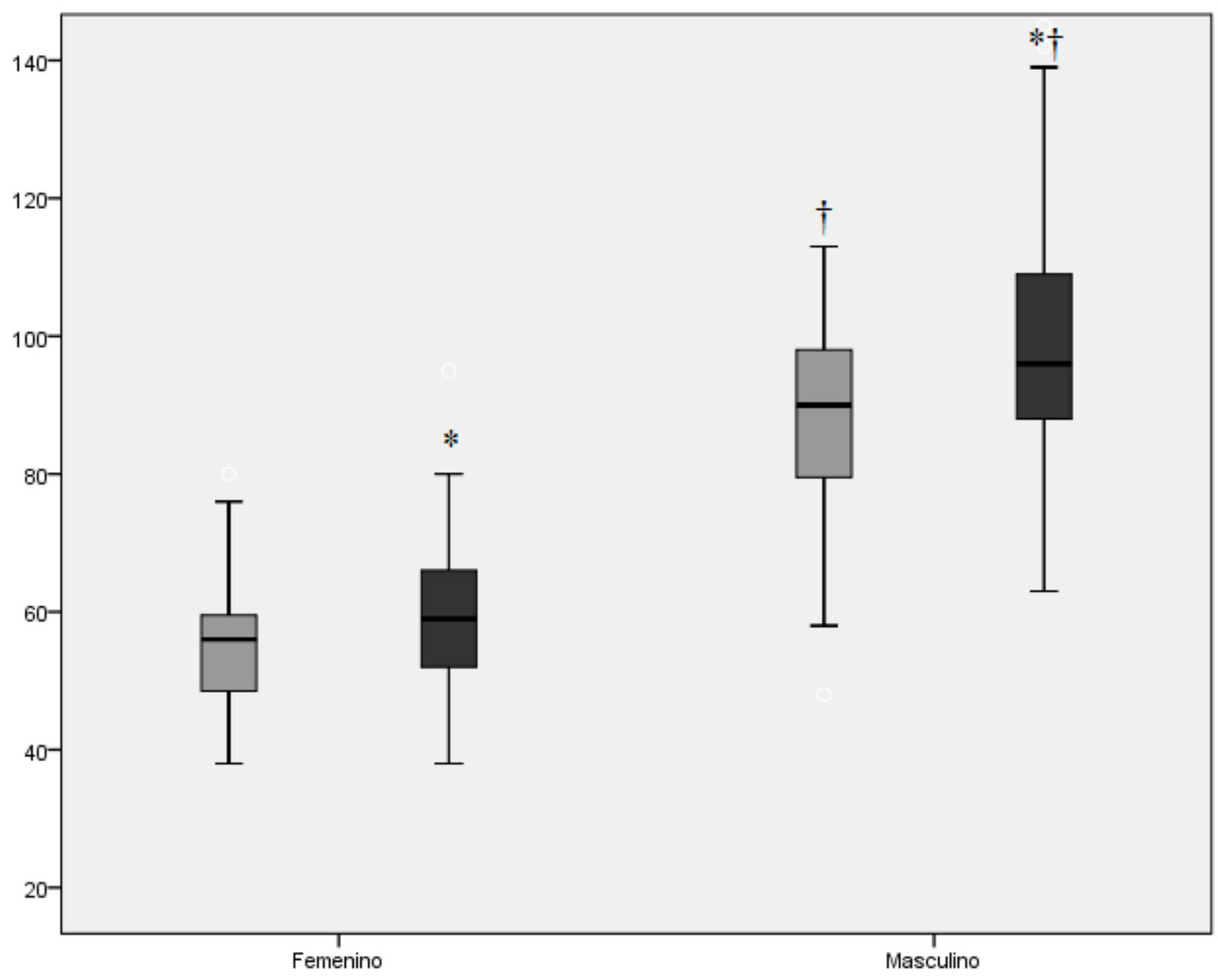

Cajas claras 18 a 19,9 años de edad, cajas oscuras 20 a 29,9 años de edad. ${ }^{*}$ Diferencias significativas con mismo sexo 18 a 19,9 años $(p<0,05)$; †diferencias significativas con mujeres de la misma edad $(p<0,05)$ Fuente: elaboración propia. 
FIGURA 4

Distribución de resultados en la prueba de Extensiones de brazos en 30 seg (repeticiones) según sexo y edad

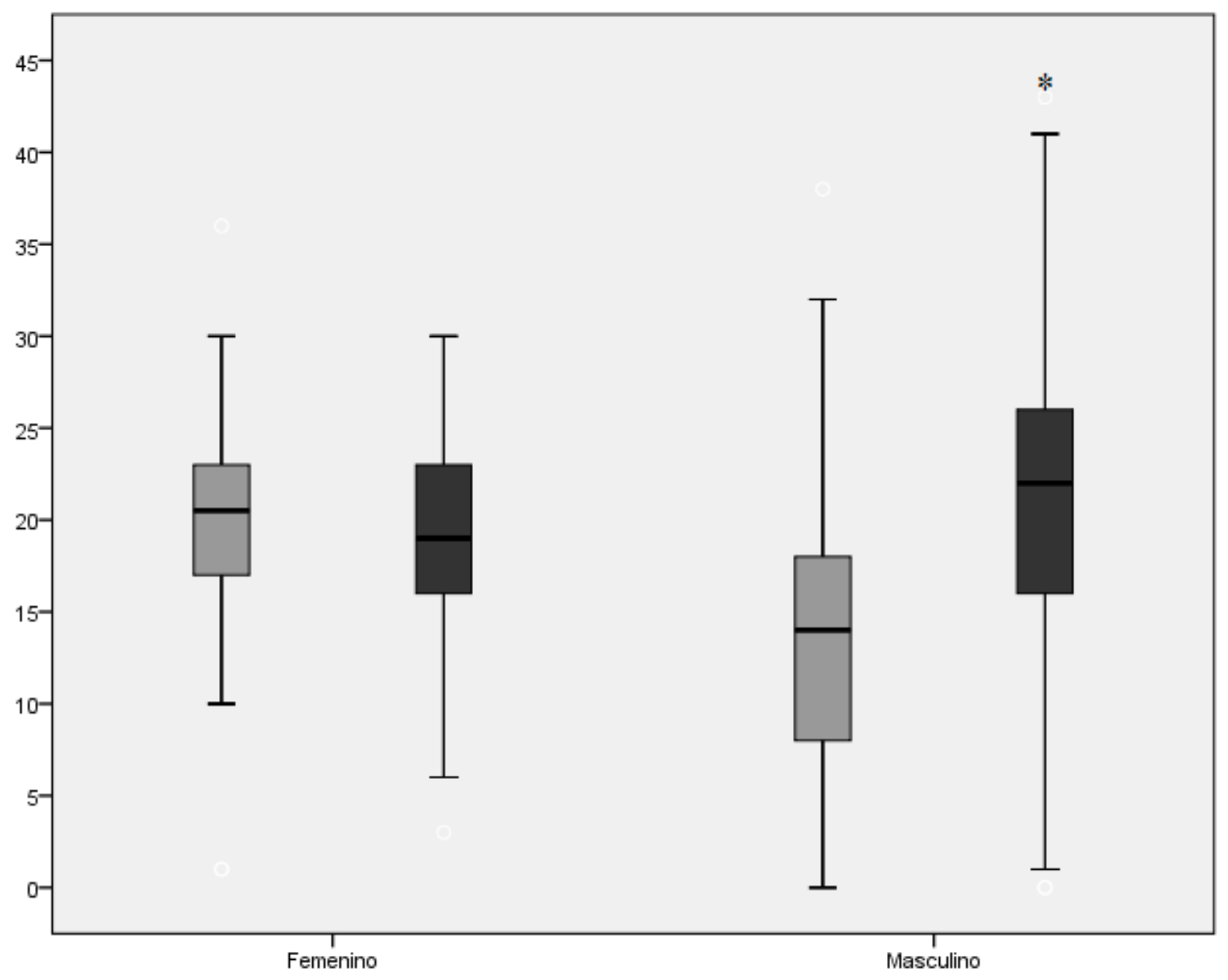

Cajas claras 18 a 19,9 años de edad, cajas oscuras 20 a 29,9 años de edad. ${ }^{*}$ Diferencias significativas con mismo sexo 18 a 19,9 años $(\mathrm{p}<0,05)$. Las diferencias entre sexos no se testearon porque realizan diferentes protocolos (ver texto) Fuente: elaboración propia. 
FIGURA 5

Distribución de resultados en la prueba de Salto horizontal $(\mathrm{cm})$ según sexo y edad

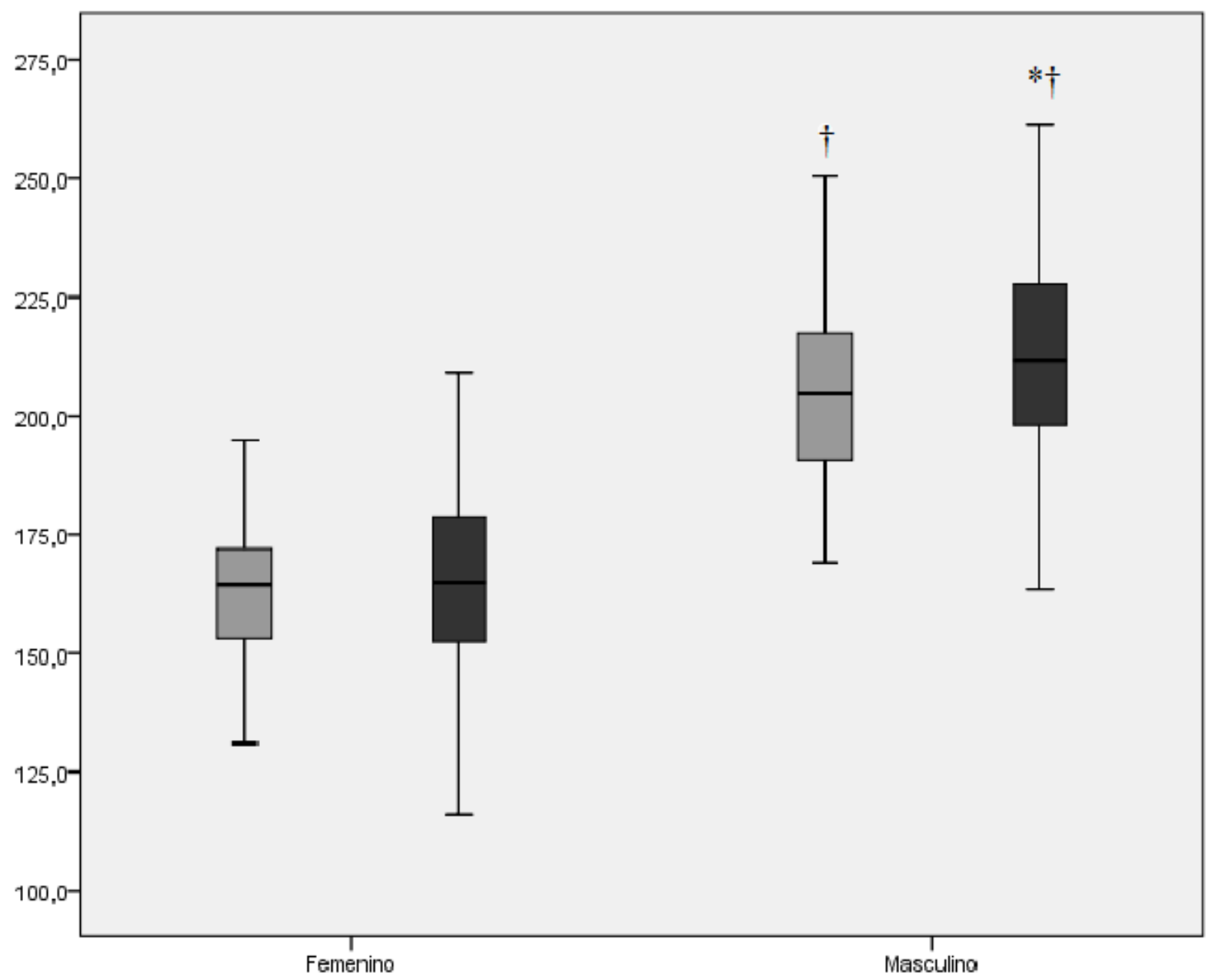

Cajas claras 18 a 19,9 años de edad, cajas oscuras 20 a 29,9 años de edad. *Diferencias significativas con mismo sexo 18 a 19,9 años $(\mathrm{p}<0,05)$; †diferencias significativas con mujeres de la misma edad $(\mathrm{p}<0,05$ Fuente: elaboración propia.

\section{TABLA 2}

Valores normativos de referencia para la prueba de Ir y volver $20 \mathrm{~m}$ según sexo y edad palieres completados

\begin{tabular}{lllll} 
& $\begin{array}{l}\text { Mujeres } \\
18-19 \\
\text { años } \\
n=61\end{array}$ & $\begin{array}{l}\text { 20-29 } \\
\text { años } \\
n=90\end{array}$ & $\begin{array}{l}\text { Varones } \\
\text { años } \\
n=99\end{array}$ & $\begin{array}{l}\text { años } \\
n=142\end{array}$ \\
\hline $\begin{array}{l}\text { Promedio } \\
\text { Desvio }\end{array}$ & 5,9 & 5,6 & 9,4 & 9,2 \\
estándar & 1,3 & 1,4 & 1,5 & 1,5 \\
\hline Percentil 5 & 3,5 & 3,0 & 6,5 & 6,6 \\
Percentil 10 & 4,5 & 4,0 & 7,5 & 7,0 \\
Percentil 25 & 5,0 & 5,0 & 8,5 & 8,0 \\
Percentil 50 & 6,0 & 5,5 & 9,5 & 9,3 \\
Percentil 75 & 7,0 & 6,5 & 10,5 & 10,5 \\
Percentil 90 & 7,5 & 7,5 & 11,5 & 11,0 \\
Percentil 95 & 8,5 & 8,2 & 12,0 & 11,5 \\
\hline
\end{tabular}


TABLA 3

Valores normativos de referencia para la prueba de Flexión de tronco al frente modificado según sexo y edad $(\mathrm{cm})$

\begin{tabular}{lllll} 
& $\begin{array}{l}\text { Mujeres } \\
18-19 \\
\text { años } \\
n=61\end{array}$ & $\begin{array}{l}\text { 20-29 } \\
\text { años } \\
n=87\end{array}$ & $\begin{array}{l}\text { Varones } \\
\text { años } \\
n=66\end{array}$ & $\begin{array}{l}20-29 \\
\text { años } \\
n=128\end{array}$ \\
\hline $\begin{array}{l}\text { Promedio } \\
\text { Desvio }\end{array}$ & 42,9 & 40,0 & 42,0 & 38,8 \\
estándar & 6,1 & 7,2 & 6,9 & 7,8 \\
\hline $\begin{array}{l}\text { Percentil 5 } \\
\text { Percentil 10 }\end{array}$ & 30,2 & 26,0 & 28,5 & 24,0 \\
Percentil 25 & 39,5 & 30,0 & 33,7 & 29,0 \\
Percentil 50 & 44,0 & 35,0 & 37,8 & 34,0 \\
Percentil 75 & 47,5 & 45,0 & 43,0 & 38,8 \\
Percentil 90 & 50,8 & 48,0 & 47,0 & 45,0 \\
Percentil 95 & 51,9 & 50,9 & 50,0 & 50,0 \\
\hline
\end{tabular}

\section{TABLA 4}

Valores normativos de referencia para la prueba de Prensión manual según sexo y edad $\left(\mathrm{kg}^{*}\right)$

\begin{tabular}{lllll} 
& $\begin{array}{l}\text { Mujeres } \\
18-19 \\
\text { años } \\
\mathrm{n}=51\end{array}$ & $\begin{array}{l}20-29 \\
\text { años } \\
\mathrm{n}=118\end{array}$ & $\begin{array}{l}\text { Varones } \\
\text { años } \\
\mathrm{n}=76\end{array}$ & $\begin{array}{l}20-29 \\
\text { años } \\
\mathrm{n}=165\end{array}$ \\
\hline $\begin{array}{l}\text { Promedio } \\
\text { Desví }\end{array}$ & 55,6 & 59,6 & 88,2 & 98,2 \\
estándar & 9,4 & 9,8 & 14,5 & 16,4 \\
\hline Percentil 5 & 42,6 & 44,0 & 63,7 & 70,3 \\
Percentil 10 & 44,0 & 47,0 & 66,7 & 77,6 \\
Percentil 25 & 48,0 & 52,0 & 79,3 & 88,0 \\
Percentil 50 & 56,0 & 59,0 & 90,0 & 96,0 \\
Percentil 75 & 60,0 & 66,0 & 98,0 & 109,5 \\
Percentil 90 & 70,0 & 74,0 & 107,6 & 120,0 \\
Percentil 95 & 73,6 & 76,1 & 112,0 & 127,0 \\
\hline "Los valores muestran la suma de ambas manos.
\end{tabular}

"Los valores muestran la suma de ambas manos.

${ }^{*}$ Los valores muestran la suma de ambas manos. 
TABLA 5

Valores normativos de referencia para la prueba de Extensiones de brazos según sexo y edad (repeticiones)

\begin{tabular}{lllll} 
& $\begin{array}{l}\text { Mujeres } \\
18-19 \\
\text { años } \\
\mathrm{n}=78\end{array}$ & $\begin{array}{l}20-29 \\
\text { años } \\
\mathrm{n}=104\end{array}$ & $\begin{array}{l}\text { Varones } \\
18-19 \\
\text { años } \\
\mathrm{n}=65\end{array}$ & $\begin{array}{l}20-29 \\
\text { años } \\
\mathrm{n}=142\end{array}$ \\
\hline $\begin{array}{l}\text { Promedio } \\
\text { Desvio }\end{array}$ & 19,7 & 19,3 & 13,4 & 21,0 \\
estándar & 5,6 & 5,2 & 8,7 & 8,3 \\
\hline Percentil 5 & 10,0 & 9,3 & 0,0 & 2,3 \\
Percentil 10 & 12,8 & 12,0 & 1,0 & 10,3 \\
Percentil 25 & 17,0 & 16,0 & 8,0 & 16,0 \\
Percentil 50 & 20,5 & 19,0 & 14,0 & 22,0 \\
Percentil 75 & 23,0 & 23,0 & 18,0 & 27,0 \\
Percentil 90 & 25,0 & 25,5 & 25,8 & 31,0 \\
Percentil 95 & 27,1 & 28,0 & 29,0 & 33,9 \\
\hline
\end{tabular}

TABLA 6

Valores normativos de referencia para la prueba de Salto horizontal según sexo y edad $(\mathrm{cm})$

\begin{tabular}{lllll} 
& $\begin{array}{l}\text { Mujeres } \\
18-19 \\
\text { años } \\
n=51\end{array}$ & $\begin{array}{l}20-29 \\
\text { años } \\
n=83\end{array}$ & $\begin{array}{l}\text { Varones } \\
\text { años } \\
n=86\end{array}$ & $\begin{array}{l}20-29 \\
\text { años } \\
n=142\end{array}$ \\
\hline $\begin{array}{l}\text { Promedio } \\
\text { Desvio }\end{array}$ & 164,1 & 164,3 & 205,7 & 213,3 \\
estándar & 15,0 & 18,4 & 19,6 & 21,6 \\
\hline Percentil 5 & 135,6 & 133,8 & 176,5 & 179,6 \\
Percentil 10 & 147,1 & 142,4 & 181,8 & 185,7 \\
Percentil 25 & 153,0 & 152,5 & 190,0 & 198,0 \\
Percentil 50 & 164,5 & 165,0 & 204,8 & 211,8 \\
Percentil 75 & 173,0 & 179,0 & 217,6 & 227,4 \\
Percentil 90 & 188,4 & 189,6 & 231,7 & 244,5 \\
Percentil 95 & 193,0 & 192,6 & 245,9 & 251,9 \\
\hline
\end{tabular}

En cuanto a la evidencia de precisión una excepción es la prueba de Extensiones de brazos en 30 seg la cual se encuentra pendiente de validación, al menos en adultos. Sin embargo hemos decidido incorporarla debido a su frecuente uso, a su alta practicidad, a los altos índices de confiabilidad encontrados y a que pruebas similares han demostrado ser suficientemente válidas (Baumgartner et al., 2002) y han sido incorporadas a baterías internacionales (CSEP, 2013; Kaminsky, 2010). Por otro lado la prueba de Ir y volver $20 \mathrm{~m}$ para evaluar el componente cardiorrespiratorio no se ha visto que sea utilizada en las baterías de adultos de población general revisadas aquí. Esto puede deberse a que su intensidad mínima implica la carrera $(8,5 \mathrm{~km} / \mathrm{h})$ y ésta puede ser una intensidad demasiado alta para sujetos adultos de baja condición física. Por este motivo suelen proponerse para esta población pruebas cardiorrespiratorias alternativas que puedan realizarse caminando (Rodríguez et al., 1998a; Kaminsky, 2010; Suni et al., 2009; CSEP, 2013). En este sentido hemos optado por proponer dos pruebas cardiorrespiratorias en la Batería Dickens, la prueba de Ir y volver 20 m que puede utilizarse en sujetos más acondicionados y la prueba de caminar 1 milla (prueba de Rockport) para sujetos de menor condición física que será incorporada en futuras versiones de la Batería Dickens. Por el contrario, la prueba de Prensión manual no se ha visto que sea utilizada con anterioridad en baterías argentinas, pero sí se propone su utilización en todas las baterías internacionales aquí revisadas; por este motivo hemos decidido incorporarla. Por último, la prueba Salto horizontal que forma parte de Batería Dickens no es utilizada en 
las baterías revisadas aquí, la cuales utilizan Salto vertical. No obstante hemos optado por Salto horizontal debido a que es igualmente precisa, pero cuenta con antecedentes de uso en Argentina y con una practicidad superior (no se necesita de un saltómetro o en su defecto de realizar dos marcas en una pared o pizarra elevada).

Los resultados de las pruebas fueron los esperados en cuanto a las diferencias por sexo. Los varones suelen contar con mayores valores de fuerza y de aptitud cardiorrespiratoria que las mujeres, quienes a su vez suelen contar con niveles iguales o levemente superiores de flexibilidad que los varones, al menos en lo que respecta a la prueba de flexibilidad utilizada en Batería Dickens (Hoeger \& Hoeger, 2010; Rodríguez et al., 1998b).

Con respecto a los valores de referencia, y como el objetivo de esta batería es que pueda utilizarse en población general (es decir en sujetos aparentemente sanos y no especializados en ninguna capacidad física o deporte), estos valores deben ser aquellos que efectivamente presente este tipo de población. Para resolver esto se sugieren realizar estudios descriptivos de una muestra representativa de sujetos de estas características en una región en particular (Rodríguez et al., 1998b). En Argentina hubo intentos de realizar este tipo de estudios pero hasta nuestro conocimiento solamente se concretó en sujetos menores de 18 años de edad (Aquila Dumit, 2012; Arcuri \& Spotorno, 1995; Barbieri, 1997; Secchi et al., 2014) y sólo en dos de estas ocasiones se realizaron muestreos representativos de las regiones estudiadas (Aquila Dumit, 2012; Barbieri, 1997). No obstante esta propuesta metodológica hay que destacar que en Argentina (y en otros países) la mayoría de los adultos de población general cuenta con niveles de actividad física bajos (INDEC, 2019) y se sabe que niveles bajos de actividad física no son los históricos de la humanidad (Chakravarthy \& Booth, 2003; Farinola, 2006), se relacionan con un perfil de salud desfavorable (Bouchard et al., 2007; Buford \& Manini, 2010) y además se relacionan con un bajo nivel de condición física (Church, Earnest, Skinner \& Blair, 2007; Garber et al., 2011). Este sesgo hacia la baja actividad física en la población general ha hecho que se ponga en duda si efectivamente puede utilizarse a esta población como referencia, ya que la condición de sedentario o insuficientemente activo lleva el funcionamiento orgánico hacia lo no saludable y fuera del estado físico evolutivamente esperable para un ser humano (Booth, Chakravarthy, Gordon \& Spangenburg, 2002; Chakravarthy \& Booth, 2004). Esto ha llevado a que se reconsidere la incorporación de sujetos de población general con estilo de vida hipocinético como grupo control en las investigaciones experimentales (Buford \& Manini, 2010). Por este motivo, siguiendo a Booth y Lees (2006), sostenemos que los valores de condición física con los que efectivamente cuenta la población general en la actualidad pueden no ser los valores de referencia de condición física más adecuados para la comparación. En lugar de esto, los valores de referencia más adecuados deberían ser aquellos valores que expresen los sujetos saludablemente activos pero que al mismo tiempo no cuenten con niveles extremadamente elevados de actividad física. De esta línea argumental se desprende que los estudiantes de la carrera de educación física pueden conformar una población de referencia más adecuada para la condición física que la población general (Booth \& Lees, 2006), siempre y cuando este grupo presente niveles de actividad física saludables. Efectivamente, según estudios realizados en la ciudad de Buenos Aires (incluido el instituto Dickens) y el Conurbano Bonaerense, la amplia mayoría de estudiantes de profesorado en educación física, aunque no todos ellos, alcanza niveles de actividad física saludables según los estándares internacionales, siendo estos niveles superiores a los de estudiantes de otras carreras que cuentan con características más similares a las de la población general (Farinola \& Bazán, 2010; Farinola, Bazán, Laíno \& Santamaría, 2012; Farinola, Polo, La Valle \& Arcuri, 2009; Pérez Ugidos, Laíño, Zelarayán \& Márquez, 2014).

En cuanto a la edad de los sujetos que forman parte de la muestra de referencia aquí propuesta hemos decidido comenzar a publicar los resultados de la franja etaria 18 a 29,9 años por ser la de mayor accesibilidad para este equipo de investigación, dejando para trabajos futuros las edades superiores. En esta franja etaria se formaron dos grupos de edad, esto es 18 a 19,9 años y 20 a 29,9 años. El punto de corte en 20 años se tomó por tres motivos. Por un lado esta división por décadas de vida es la que observamos que se utiliza más frecuentemente en las baterías internacionales para adultos de población general (por ejemplo en Pescatello, 
2014; Suni et al., 2009; CSEP, 2013); por otro lado fue la división de edades en la que nuestra muestra quedó mejor balanceada en cuanto a la cantidad de sujetos contenidos en cada categoría; y por último porque al realizar esta división encontramos diferencias significativas en mayor cantidad de pruebas cuando testeamos las diferencias entre los grupos de edad (ver figuras 1 a 5 ) que con otros puntos de corte (esto es 18 a 24,9 y 25 a 29,9; datos no publicados). Esto quiere decir que el primer grupo etario es diferente del segundo por lo que corresponde presentar valores de referencia por separado.

Se destaca que el grado de participación de los sujetos haya sido cercano al $100 \%$, lo que permite inferir que no existe un sesgo interno y que todos los niveles de condición física que se hallan en los estudiantes de nuestra muestra están representados en las respectivas tablas.

No obstante existe una limitación a considerar, y es que esta muestra pertenece a un solo instituto de educación física de una sola ciudad del país. Por lo tanto, si lo que se busca es una muestra de referencia a nivel nacional, sería aconsejable ampliar los casos incorporando sujetos de otros institutos y de otras ciudades. Igualmente, mientras esto esté pendiente de realización, consideramos más pertinente utilizar estos valores de referencia para sujetos argentinos de población general de estas edades que las tablas extranjeras.

En el corto y mediano plazo se prevé ampliar esta batería a más dimensiones, pruebas y franjas etarias para disponer de una batería completa que permita la evaluación de la CFRS en sujetos adultos argentinos de población general.

\section{Conclusiones}

Proponer una batería de evaluación de la condición física para población general adulta es algo que las instituciones dedicadas a la promoción de la actividad física con fines de salud y bienestar han realizado en diferentes países. En Argentina no se han encontrado antecedentes de este tipo de instrumentos para dicha población. La conveniencia de contar con una batería local radica en que las pruebas tienen que ser posibles de administrarse sin mayores dificultades por la mayoría de los profesionales. Para esto es indispensable que no requieran instrumentos e instalaciones de difícil adquisición en la región para la cual se pensó la batería. Por otro lado los valores de referencia, cuando se trata de evaluación normativa, deben ser los adecuados para los habitantes de dicha región. Por estos motivos no siempre conforma una buena práctica utilizar baterías construidas en países diferentes al cual se va aplicar el instrumento. En este trabajo se buscó comenzar a cubrir la vacancia que resulta de no contar con este tipo de baterías en Argentina. A tal efecto se ha realizado una propuesta concreta con fundamento en los antecedentes argentinos e internacionales y en las investigaciones y posicionamientos científicos dentro del campo. En esto radica el mayor aporte. Sin embargo esta propuesta no está exenta de dificultades que deberán ir resolviéndose. La selección de dimensiones a evaluar, creemos, no presenta mayores dificultades debido a que existe un consenso prácticamente unánime acerca de la cuestión. Una discusión algo mayor podría caber en cuanto a qué pruebas deberían formar parte de una batería para Argentina, y una discusión más profunda cabe para decidir cuál es la población de referencia más adecuada. Los fundamentos presentados en los cuales se sostienen las decisiones que se han tomado a estos respectos conforman otro aporte de este trabajo que creemos significativo para comenzar a construir una batería para la evaluación de la condición física en adultos argentinos.

\section{Agradecimientos}

A las autoridades pasadas y presentes del ISEF No2 "Prof. Federico W. Dickens", especialmente al Prof. Fernando Curci por su permanente colaboración y predisposición. A los colegas del Laboratorio de Actividad y Aptitud Física "Lic. Pedro Giorno". Y a todos los alumnos del instituto Dickens que han participado voluntariosamente para permitir que este trabajo pueda concretarse. 


\section{REFERENCIAS BIBLIOGRÁficAS}

Aquila Dumit, F. (2012). Plan Provincial de evaluación, de aspectos morfológicos, kinefilácticos y condición física, de alumnos de 10 a 18 años de edad del nivel primario, secundario y educación especial de la Provincia de Mendoza - 2011. Mendoza: Gobierno de Mendoza, Dirección de Educación Física; 88 pp. Recuperado de http://bases.mendoza.edu.ar/intranet/educfisica/Plan\%20Provincial\%20de\%20Evaluaci\%C3\%B3n\%20-\% 20RESULTADOS\%202011.pdf

Arcuri, C., y Spotorno, A. (1995). Plan de evaluación del perfil psicomotor, morfológico y físico en niños de 5 a 12 años de edad de la Ciudad de Buenos Aires. Buenos Aires: Gobierno de la Ciudad de Buenos Aires, Dirección General de Deportes; 72 pp.

Ayala, F., Sainz de Baranda, P., de Ste Croix, M., y Santonja, F. (2012). Fiabilidad y validez de las pruebas sit-and-reach: revisión sistemática. Revista Andaluza de Medicina del Deporte, 5(2), 57-66. Recuperado de http://www.elsevier.es/es-revista-revista-andaluza-medicina-del-deporte-284-articulo-fiabilidad-validez-laspruebas-sit-and-reach-X1888754612495328

Barbieri, C. (1997). PEDIAFyS: Programa de Evaluación, Diagnóstico e Investigación de la Aptitud Física y la Salud. Provincia de Buenos Aires: Instituto Bonaerense del Deporte y Dirección General de Cultura y Educación; 57 pp.

Baumgartner, T., Oh, S., Chung, H., \& Hales, D. (2002). Objectivity, reliability, and validity for a revised push-up test protocol. Measurement in Physical Education and Exercise Science, 6(4), 225-242. https://doi.org/10.1207 /S15327841MPEE0604_2

Blair, S., Kohl, H. III, Paffenbarger, R. Jr, Clark, D., Cooper, K., \& Gibbons, L. (1989). Physical fitness and all-cause mortality: a prospective study of healthy men and women.JAMA, 262(17), 2395-2401. doi: https://10.1001 / jama.1989.03430170057028

Blair, S., Kohl, H. III, Barlow, C., Paffenbarger, R. Jr, Gibbons, L., \& Macera, C. (1995). Changes in physical fitness and all-cause mortality: A prospective study of healthy and unhealthy men.JAMA, 273(14), 1093-1098. doi: h ttps://10.1001/jama.1995.03520380029031

Blázquez Sánchez, D. (1992). Evaluar en Educación Física. Barcelona: INDE.

Booth, F., Chakravarthy, M., Gordon, S., \& Spangenburg, E. (2002). Waging war on physical inactivity: using modern molecular ammunition against an ancient enemy.J Appl Physiol, 93(1), 3-30. https://doi.org/10.1152/japplph ysiol.00073.2002

Booth, F., \& Lees, S. (2006). Physically active subjects should be the control group. Medicine \& Science in Sports \& Exercise, 38(3), 405-406. doi: https://10.1249/01.mss.0000205117.11882.65

Bouchard, C., Blair, S., \& Haskell, W. (2007). Why study physical activity and health? En C. Bouchard, S. Blair, \& W. Haskell, (Eds.), Physical activity and health (pp.3-19). Champaign, IL: Human Kinetics.

Buford, T., \& Manini, T. (2010). Sedentary individuals as "controls" in human studies: The correct approach? Proc Natl Acad Sci USA, 107(34): E134. doi: https://10.1073/pnas.1008118107

Canadian Society for Exercise Physiology (CSEP). (2013). CSEP-Physical Activity Training for Health (CSEP$P A T H)$. Ottawa, ON : Canadian Society for Exercise Physiology.

Caspersen, C. J., Powell, K. E., \& Christenson, G. M. (1985). Physical activity, exercise, and physical fitness: definitions and distinctions for health-related research. Public Health Reports, 100(2), 126-131. Recuperado de https://w ww.ncbi.nlm.nih.gov/pmc/articles/PMC1424733/pdf/pubhealthrep00100-0016.pdf

Chakravarthy, M. V., \& Booth, F. W. (2003). Exercise. Philadelphia, PA: Hanley \& Belfus.

Chakravarthy, M. V., \& Booth, F. W. (2004). Eating, exercise, and "thrifty" genotypes: connecting the dots toward an evolutionary understanding of modern chronic diseases. J Appl Physiol, 96(1), 3-10. https://doi.org/10.115 2/japplphysiol.00757.2003

Church, T., Earnest, C., Skinner, J., \& Blair, S. (2007). Effects of different doses of physical activity on cardiorespiratory fitness among sedentary, overweight or obese postmenopausal women with elevated blood pressure: A randomized controlled trial.JAMA, 297(19), 2081-2091. doi: https://10.1001/jama.297.19.2081 
Delgado, D., Renda, J., Rojas, O., y Supital R. (2011). Comparación de tres prueba de aptitud física, entre los resultados obtenidos en el ingreso y durante el trayecto de la carrera, en alumnos del ISEF №1. Revista electrónica de Ciencias Aplicadas al Deporte, 4(15). Recuperado de https://studylib.es/doc/5991891/descargar-este-fichero-pdf

Farinola, M. (2006). Explicación de un modelo integrador sobre la relación de causalidad entre la actividad física, la salud y el riesgo de muerte prematura. apunts Educación Física y Deportes, 85, 15-27. Recuperado de http://ww w.revista-apunts.com/es/hemeroteca?article $=113$

Farinola, M., y Bazán, N. (2010). Niveles de actividad física en estudiantes de la carrera de profesorado universitario en educación física y de otras carreras de grado en la Universidad de Flores. Calidad de Vida \& Salud, 3(1): 25-44. Recuperado de http://revistacdvs.uflo.edu.ar/index.php/CdVUFLO/article/view/27

Farinola, M., Bazán, N., Laíño, F., y Santamaría, C. (2012). Actividad física y conducta sedentaria en estudiantes terciarios y universitarios de la Región Metropolitana de Buenos Aires (Argentina). R. bras. Ci. e Mov, 20(3), 79-90. Recuperado de https://portalrevistas.ucb.br/index.php/RBCM/article/view/3173

Farinola, M., Polo, M., La Valle, L., y Arcuri, C. (2009). Niveles de actividad física en alumnos/as del Instituto Superior de Educación Física No 2 Federico W. Dickens. Revista electrónica de Ciencias Aplicadas al Deporte, 2(5). Recuperado de https://es.calameo.com/books/005092469864bd9c75529

Garber, C., Blissmer, B., Deschenes, M., Franklin, B., Lamonte, M., Lee, I., Nieman D., \& Swain, D. (2011). Quantity and quality of exercise for developing and maintaining cardiorespiratory, musculoskeletal, and neuromotor fitness in apparently healthy adults: Guidance for prescribing exercise. Medicine \& Science in Sports \& Exercise, 43(7), 1334-1359. doi: https://10.1249/MSS.0b013e318213fefb

García López, J., y Herrero Alonso, J. (2005). Variables cinéticas de la batida relacionadas con el rendimiento del salto horizontal a pies juntos. Biomecánica, 12(2), 61-70. Recuperado de https://upcommons.upc.edu/handle/2099 $/ 115$

George, J., Fisher, A., \& Vehrs, P. (1996). Tests y pruebas fisicas. Barcelona: Paidotribo.

Hoeger, W., \& Hoeger, S. (2010). Principles and labs for physical fitness. 7th Edition. Belmont, CA: Wadsworth.

Instituto Nacional de Estadística y Censos - INDEC. (2019). $4^{\circ}$ Encuesta Nacional de Factores de Riesgo: resultados preliminares (1. ed.). Buenos Aires: INDEC, Secretaría de Gobierno de Salud. Recuperado de https://www.ind ec.gov.ar/ftp/cuadros/publicaciones/enfr_2018_resultados_preliminares.pdf

Kaminsky, L. (Ed.). (2010). ACSM's Health-related physical fitness assessment manual. 3th Edition. Philadelphia, PA: Lippincott Williams \& Wilkins

Katzmarzyk, P., Church, T., \& Blair, S. (2004). Cardiorespiratory fitness attenuates the effects of the metabolic syndrome on all-cause and cardiovascular disease mortality in men. Arch Intern Med, 164(10), 1092-1097. doi: https://10.1001/archinte.164.10.1092

Kenney, W., Wilmore, J., \& Costill, D. (2012). Physiology of sport and exercise. 5th ed. Champaign, IL: Human Kinetics.

Lauretani, F., Russo, C., Bandinelli, S., Bartali, B., Cavazzini, C., Di Iorio, A., Corsi, A., Rantanen, T., Guralnik, J., \& Ferrucci, L. (2003). Age-associated changes in skeletal muscles and their effect on mobility: an operational diagnosis of sarcopenia.J Appl Physiol, 95(5), 1851-1860. https://doi.org/10.1152/japplphysiol.00246.2003

Léger, L., Mercier, D., Gadoury, C., \& Lambert, J. (1988). The Multistage 20 Metre Shuttle Run test for aerobic fitness. J Sports Sci, 6(2), 93-101. https://doi.org/10.1080/02640418808729800

López Elvira, J., Grande Rodríguez, I., Meana Riera, M., y Aguado Jódar, X. (1999). Análisis de la reproductibilidad en tres tests de salto con plataforma de fuerzas y de contactos. apunts Educación Física y Deportes, 58, 62-66. Recuperado de http://www.revista-apunts.com/es/hemeroteca?article $=479$

Mathiowetz, V. (2002). Comparison of Rolyan and Jamar dynamometers for measuring grip strength. Occup Ther Int, 9(3), 201-209. https://doi.org/10.1002/oti.165

Mayorga-Vega, D., Merino-Marban, R., \& Viciana, J. (2014).Criterion-related validity of Sit and Reach Test for estimating hamstring and lumbar extensibility: A meta analysis. Journal of Sports Medicine, 13(1), 1-14. Recuperado de https://www.jssm.org/hf.php?id=jssm-13-1.xml 
Ministerio de Salud. (2014). Indicadores básicos 2014. Buenos Aires: Ministerio de Salud de la Nación, Argentina. Recuperado de http://publicaciones.ops.org.ar/publicaciones/indicadores/Indicadores2014.pdf

Pangrazi, R, \& Hastad, D. (1989). Fitness in the elementary schools (2nd ed.). Reston, VA: AAHPERD.

Paradisis, G., Zacharogiannis, E., Mandila, D., Smirtiotou, A., Argeitaki, P., \& Cooke, C. (2014). Multi-Stage 20$\mathrm{m}$ Shuttle Run fitness test, maximal oxygen uptake and velocity at maximal oxygen uptake. Journal of Human Kinetics, 41(1), 81-87. Recuperado de https://content.sciendo.com/view/journals/hukin/41/1/article-p81.xml

Pate, R. (1988). The evolving definition of physical fitness. Quest, 40(3), 174-179. https://doi.org/10.1080/003362 97.1988.10483898

Pérez Ugidos, G., Laíño, F., Zelarayán, J., y Márquez, S. (2014). Actividad física y hábitos de salud en estudiantes universitarios argentinos. Nutr Hosp, 30(4), 896-904. http://dx.doi.org/10.3305/nh.2014.30.4.7641

Pescatello, L. (Senior editor). (2014). ACSM'S Guidelines for exercise testing and prescription. 9th edition. Philadelphia, PA: Thompson Wolters Kluwer/Lippincott Williams \& Wilkins.

Roberts, H., Denison, H., Martin, H., Patel, H., Syddall, H., Cooper, C., \& Sayer, A. (2011). A review of the measurement of grip strength in clinical and epidemiological studies: towards a standardised approach. Age and Ageing, 40(4), 423-429. doi: https://10.1093/ageing/afr051

Rode, A., \& Shephard, R. J. (1994). Physiological consequences of acculturation: a 20-year study of fitness in an Inuit community. Eur J Appl Physiol, 69(6), 516-524. https://doi.org/10.1007/BF00239869

Rodríguez, F., Gusi, N., Valenzuela, A., Nácher, S., Nogués, J., y Marina, M. (1998a). Valoración de la condición física saludable en adultos (I): antecedentes y protocolos de la batería AFISAL-INEFC. apunts Educación Física y Deportes, 52, 54-75. Recuperado de http://www.revista-apunts.com/es/hemeroteca?article $=674$

Rodríguez, F., Valenzuela, A., Gusi, N., Nácher, S., y Gallardo, I. (1998b). Valoración de la condición física saludable en adultos (II): fiabilidad, aplicabilidad y valores normativos de la batería AFISAL-INEFC. apunts Educación Física y Deportes, 54, 54-65. Recuperado de http://www.revista-apunts.com/es/hemeroteca?article $=648$

Rojas, E., Renda, J., Cova, F., y Mostovoi, G. (2010). Estudio preliminar para la comparación de la resistencia general de los alumnos del ISEF $N^{\circ} 1$ entre el test realizado en el ingreso y durante el trayecto de la carrera. Revista electrónica de Ciencias Aplicadas al Deporte, 3(11). Recuperado de https://docplayer.es/28800881-Revista-electronica-deciencias-aplicadas-al-deporte-vol-3-n-11-buenos-aires-12-2010.html

Secchi, J., García, G., España-Romero, V., y Castro-Piñero, J. (2014). Condición física y riesgo cardiovascular futuro en niños y adolescentes argentinos: una introducción de la batería ALPHA. Arch Argent Pediatr, 112(2), 132-140. Recuperado de https://www.sap.org.ar/docs/publicaciones/archivosarg/2014/v112n2a05.pdf

Smith, J., Eather, N., Morgan, P., Plotnikoff, R., Faigenbaum, A., \& Lubans, D. (2014). The health benefits of muscular fitness for children and adolescents: A systematic review and meta-analysis. Sports Medicine, 44(9), 1209-1223. doi: https://10.1007/s40279-014-0196-4

Suni, J., Marjo, P., \& Husu, R. (2009). Fitness for Health: The ALPHA-FIT test battery for adults aged 18-69. UKK Institute for Health Promotion Research, Tampere, Finland. Recuperado de http://www.ukkinstituutti.fi/file bank/500-ALPHA_FIT_Testers_Manual.pdf

Vásquez-Gómez, J., Castillo-Retamal, M., Souza de Carvalho, R., Faundez-Casanova, C., \& Torrealba-Campos, A. (2018). Antropometría, nivel de actividad física y condición física en estudiantes de educación física tras cuatro años en la universidad. Nutr Clín Diet Hosp, 38(1), 160-164. Recuperado de http://revista.nutricion.org/PDF /JVASQUEZ.pdf

Warburton, D., Gledhill, N., \& Quinney, A. (2001). Musculoskeletal fitness and health. Can J Appl Physiol, 26(2), 217-237. https://doi.org/10.1139/h01-013

Warburton, D., Nicol, C., \& Bredin, S. (2006). Health benefits of physical activity: the evidence. Can Med Assoc J, 174(6), 801-809. doi: https://10.1503/cmaj.051351

\section{BY-NC-SA}

